\title{
Management strategies and cost effectiveness in the prevention of cervical cancer
}

This article was published in the following Dove Press journal:

ClinicoEconomics and Outcomes Research

3 June 2009

Number of times this article has been viewed

\section{Smita R Prasad \\ University of Kentucky, College of Public Health, Lexington, KY, USA}

Correspondence: Smita R Prasad University of Kentucky, College of Public Health, Health Services Management, I2 I Washington Ave., Room 103, Lexington, KY 40536, USA

$\mathrm{Tel}+\mathrm{I}$ 859-327-4236

Email smita.prasad@gmail.com
Abstract: This paper introduces the burden of cervical cancer and the primary and secondary preventative interventions currently available, and provides an overview of the bivalent and quadrivalent human papillomavirus (HPV) vaccines. A brief account of the virology and the clinical efficacy of both is given. Examinations of the two main types of cost analyses, cost-effective and cost-benefit, as well as examples, are presented in addition to the possible impact these analyses and further economic models will have on budget and policy making decisions. In general, most cost analyses provide support for the implementation of primary prevention strategies, HPV immunization, in conjunction with cervical cancer screening programs. Vaccination against HPV, therefore, is a cost-effective cervical cancer prevention mechanism. Keywords: cervical cancer, human papillomavirus, HPV

\section{Introduction}

Cervical cancer is the second most common cause of cancer death in adult females. In developing nations where the barriers to access of are the highest, it is frequently the most common cancer in women because these areas tend to be underserved and resource-poor. ${ }^{1,2}$ Cervical cancer incidence and mortality rates vary globally, a function of ethnicity, demography, geography, socioeconomic status, and access to healthcare. In the United States (US) and many other industrialized nations, because of the increased screening, cervical cancer incidence and mortality rates have been declining for decades. ${ }^{3}$ Yet even in developed countries, 1 out of 125 women ( 0 to 64 years) still have an accumulated lifetime risk of acquiring the disease. ${ }^{4}$ Despite notably reduced incidence and mortality rates, the burden of cervical cancer precursor lesions and the disease is substantial to both social and health care paradigms.

The human papillomavirus (HPV) is the only recognized carcinogen that is a "necessary cause" of cancer in humans. ${ }^{5,6}$ It is sexually transmitted and high-risk types of HPV have been linked to cancers of the cervix, penis, vulva, vagina, anal canal, and perianal skin. ${ }^{5,7}$ Without intervention or treatment, most HPV infections will clear the body naturally. However, should persistent infection of HPV occur, the risk for cervical and other HPV-associated cancers increases. ${ }^{6}$ In the US alone, HPV types 16 and 18 result in approximately $30 \%$ of vaginal and vulvar cancers, $70 \%$ of cervical cancer, and $80 \%$ of anal cancer. ${ }^{8-12}$ More than $90 \%$ of anogenital warts in the US are attributable to HPV types 6 and 11. ${ }^{12,13}$ The total direct and indirect economic costs associated with HPV-related genital warts as well as screening for and treating cervical cancer in the United States could be as much as US\$4 billion each year. ${ }^{8,14,15}$ Eliminating these burdens necessitates inexpensive, efficient, and effective interventions. 
Cervical cancer accounts for approximately $10 \%$ of all cancers, not including non-melanoma skin cancers. It is a leading cause of morbidity and mortality afflicting women and is preventable. ${ }^{20}$ In 2006, the FDA approved a quadrivalent HPV vaccine (Gardasil ${ }^{\circledR}$; Merck \& Co. Inc., White House Station, NJ, USA). This vaccine targets the high-risk types of the virus that are the most significant contributors to cervical cancer, types 16 and 18, which cause approximately $70 \%$ of all cervical cancer cases, and the two low-risk types most associated with genital warts, types 6 and 11. ${ }^{16,21}$ A second prophylactic vaccine (Cervarix ${ }^{\circledR}$; GlaxoSmithKline Biologicals, Rixensart, Belgium) is bivalent and targets HPV types 16 and 18. Licensed for use in Europe but awaiting approval from the Food and Drug Administration in the US, the bivalent vaccine still shows promise as an immunization and an opportunity for preventative care against cervical cancer.

Early detection, diagnosis, and treatment of cervical cancer prelesions and neoplasia, is the most important factor in designing and implementing cervical cancer screening programs. ${ }^{18}$ With the advent of quadrivalent and bivalent prophylactic vaccines that target the two high-risk types of HPV, types 16 and 18, that can cause cervical cancer should persistent infection occur, there is new hope for a primary prevention strategy coupled with pre-existing screening programs, to eradicate cervical cancer and other diseases associated with HPV and to alleviate the financial burden.

This review will examine management and cost effectiveness strategies presently employed in cervical cancer prevention including primary and secondary interventions. Elucidation of the effects of these interventions on cost analyses, budget impacting, and modeling as well as implications to healthcare and public health policy will serve to provide information on the methods that will be the most successful in the quest to reduce cervical cancer incidence and mortality in the future.

\section{Primary and secondary prevention interventions}

The implementation of screening programs is the secondary prevention strategy instrumental in reducing cervical cancer incidence and mortality rates in the 20th century. In unscreened populations alone, introduction of screening programs has demonstrated significant reductions in cervical cancer rates by $60 \%$ to $90 \%$ within 3 years of screening instigation. ${ }^{19}$ In conjunction with the Pap smear and other technological advancements such as liquid-based cytology, HPV DNA testing, and computer-assisted smear-reading, some countries have seen drastic reductions in cervical cancer mortality rates by as much as $75 \%$ since these tactics were introduced over 50 years ago. ${ }^{20,21}$ Secondary prevention has thus played a pivotal role in diminishing cervical cancer mortality rates.

Traditionally, cervical cancer screening programs have utilized cervical cytology as a means of detecting and diagnosing cervical cancer and precancerous lesions. Despite these interventions, cervical cancer incidence and mortality rates and the economic burden affiliated with this disease, remain high. In developing nations and rural areas of industrialized countries where barriers to access of care are evident, this may be due to implementation and compliance issues of cervical cancer screening programs. ${ }^{9,22}$ Developing cervical cancer screening programs requires consideration of many issues and parameters. These include the age interval at which screening should occur and new technological advancements that enhance and can combine with existing methods of cytology. ${ }^{22,23}$

Cervical cancer is preventable because the progression from preneoplasia to invasive cancer is gradual, which affords ample time for detection and treatment. Furthermore, it is preventable because the methodology and strategy, namely repetitive screening and Pap Smears, needed to detect cervical premalignancy are available. Cervical cytology, however, is not without imperfections. Approximately $30 \%$ of all cervical cancers result from subpar sensitivity. ${ }^{24}$ Moreover, cytologic screening has less than optimal sensitivity, equivocal results, and restrained reproducibility. ${ }^{1,25,26}$ As a result, noncytologybased screening programs are evolving that employ HPV DNA testing or visual inspection with acetic acid followed by treatment using cryotherapy. These alternatives are cheaper than cytology and are equally as reliable. In resource-poor areas where colposcopy and histopathological services are not readily available, these options are attractive. ${ }^{28}$ In addition, HPV DNA testing shows greater sensitivity, as much as $90 \%$, in many screening trials, and may therefore be a more optimal secondary prevention method. ${ }^{29}$

Screening alone is not sufficient to maximize cervical cancer prevention. The HPV vaccination is a primary prevention strategy that mandates investigation and possible incorporation into existing secondary screening programs. The American Cancer Society has stipulated guidelines for the use of the HPV vaccine as a preventative method, recommending that public health and policy endeavors guarantee access to and promote the prophylaxis. More importantly, efforts should encourage, through education, all females of every racial, ethnic, and socioeconomic group of the 
appropriate age to receive the vaccination, especially those females most affected by the burden of cervical cancer. ${ }^{5}$

\section{Overview of vaccines, virology, and clinical efficacy}

Most HPV infections generally educe local cellular immunity and clear the body naturally. However, research suggests that the response elicited by antibodies produced by natural HPV infection may be limited in the body although they seem to protect against repeat infection of the same type of HPV. The two prophylactic vaccines commercially available that target HPV and utilize L1 structural capsular proteins may confer longer lasting immunity. The proteins derive from genetic recombination of Saccharomyces cervisiae, in the case of the quadrivalent vaccine, or baculovirus, in the case of the bivalent vaccine, and resemble real HPV antigenically in their ability to form virus-like particles (VLPs) and self-assemble. ${ }^{2,29}$ A strong, persistent, type-specific response follows vaccination of HPV VLPs, which do not comprise attenuated or live virus. ${ }^{2,30,31}$

Both vaccines are highly efficacious. Three studies, a phase II study among females aged 16 to 23 years of age and two phase III studies, one among females aged 16 to 23 years and the other females aged 16 to 26 years, have been conducted that evaluate the efficacy of the quadrivalent HPV vaccine. ${ }^{32-34}$ From these studies, the determined efficacy of the quadrivalent vaccine was extremely high against persistent infection by HPV types $6,11,16$, and 18, against low-grade cervical intraepithelial neoplasia (CIN) and adenocarcinoma in situ. Additional studies have indicated that after 3 years, the quadrivalent vaccine specifically has an $86 \%$ efficacy in combating type 16 infection $(P<0.0001), 89 \%$ efficacy against type $18(P=0.0103)$, and an $89 \%$ efficacy for the prevention of persistent cervical infection of all four types $(P<0.0001))^{32,35}$

The efficacy of the bivalent HPV vaccine needs further elucidation. Fewer studies have examined the efficacy of this vaccine most likely because it is younger and does not have worldwide recognition and licensure. The published literature, however, indicates that the bivalent vaccine is highly efficacious against persistent infection by HPV types 16 and 18. Phase II and phase III trials of the bivalent vaccine demonstrated efficacious success in preventing high-grade $\mathrm{CIN}$ and cervical cancer that were causally associated to HPV types 16 and 18 in addition to preventing persistent infection of the two types. ${ }^{36,37}$ Another study found the bivalent vaccine to have $100 \%$ (95\% CI, $42.4 \%$ to $100 \%$ ) efficacy in preventing CIN related to HPV types 16 and 18 among women 15 to 25 years of age that completed the 3-dose vaccination regimen and participated in a follow-up study for 44 to 53 months. ${ }^{38}$

It remains unknown whether boosters will be necessary in the future for persistent immunity or long-term efficacy and it will be years before the ascertainment of the definitiveness of the vaccine in preventing cervical cancer later in life. Nevertheless, the vaccine shows unprecedented promise in combating cervical cancer by eliminating persistent HPV infection. ${ }^{17}$

\section{Comparative review of cost analyses}

The costs of treating cervical cancer are not likely to remain constant especially in countries where the population is aging and the life expectancy is increasing with accompanying risks of cancer and other chronic diseases. ${ }^{3,39}$ Positive indirect costs such as time and travel costs should reasonably rise as well as the costs to treat cervical precancer and invasive cervical cancer side effects. ${ }^{3,40}$ Since detection and diagnoses of cervical diseases occurs at different stages of cancer, an exact monetary value of treating cervical cancer is impossible to quantify. Treatment options and costs are a function of the cancer stage, and costs rise as the severity of diagnosis increases and can vary by age. ${ }^{3,41}$ Cost analyses, therefore, attempt to discern the effects of implementing cervical cancer prevention programs that incorporate a primary prevention mechanism, the HPV vaccine, on the costs associated with cervical cancer and its sequela.

Most economic evaluations conducted and relevant to the study of the HPV vaccine are cost-effectiveness analyses and not cost-benefit analyses. ${ }^{42-44}$ Despite the fact that both are instruments used to judge the efficiency of health services programs, there is a substantial difference between the two types of analyses. While both are important for decision-making in planning, implementing, continuing, and expanding health service programs and both analyses assist in identification and comparability of either known or expected costs with actual or anticipated benefits, the primary difference is in the manner of expression of the outcomes or effects of a program. Cost-benefit analyses attribute monetary value to both costs and benefits, which at times can be difficult to assess. Cost-effectiveness analyses, on the other hand, only monetize the costs of a program and not the benefits. Benefits in a cost-effectiveness analysis, usually expressed as program outcomes, represent the goals of the program. Therefore, in relation to health services programs, cost-benefit analyses are primarily concerned with cost relative to output, while cost-effectiveness analyses assess goal achievement and program efficiency. ${ }^{45}$ 
It is important to note that neither cost-benefit analysis nor cost-effectiveness analysis is necessarily better. The cost-effectiveness analyses that have been conducted on potential HPV vaccine programs and existing cervical cancer screening programs all support use of the vaccine because of increased efficiency in these programs. However, these analyses are not necessarily generalizable to all cervical cancer-screening programs across the US and the rest of the world. Demographics affect the reliability of these analyses since varying populations can lead very different lifestyles. A cost-benefit analysis, therefore, could result in dramatically different conclusions for a specific region of study than these cost-effectiveness evaluations.

There is a plethora of proposed mathematical models for predicting both the economic and epidemiological impact of the HPV vaccine against HPV infection and cervical cancer. ${ }^{44}$ The three prominent types of mathematical models are cohort, population dynamic, and hybrid. The various model types all exploit common factors that affect the risk associated with acquiring and clearing infection of HPV, progression of CIN and subsequent regression, and incidence of invasive cancer. For example, the probability of acquiring HPV in simulation model of one cohort depends on present age, age of sexual debut, the type of HPV, exposure to screening and Pap smears, and whether immunity has been conferred to the specific type of HPV acquired. On the other hand, sexual contact patterns and viral distribution influence rates of HPV acquisition in transmission models. ${ }^{2}$ All the models in this area, regardless of type, however, conclude that vaccination against HPV in the end can reduce incidence and mortality of cervical cancer, but the models evaluate the cost-effectiveness of preventative vaccination strategies rather than the cost-benefit of the vaccine itself. ${ }^{6,43}$

It is not possible to calculate the cost of quality-adjusted life years (QALY) and disability-adjusted life years (DALY) without concretely defining the quantity of indirect costs, which is a necessary component of the calculation. However, a recently published report summarized four previous studies that estimated the potential cost-effectiveness of the HPV vaccine in regards to screening practices in the US. Two studies employed Markov models of the natural history of HPV infection to estimate the cost-effectiveness of vaccinating 12-year-old females. These studies, under different assumptions of vaccine efficacy and cost, estimated lifetime reductions in cervical cancer incidence in the vaccinated cohort between $20 \%$ and $58 \%$. The corresponding costs of vaccination compared to no vaccination ranged from
US\$22,800 per QALY, in 2001 dollars, to $\$ 24,300$ per QALY in 2002 dollars. ${ }^{23,42}$ The two remaining analyses based on dynamic transmission models found reductions of $62 \%$ and $75 \%$ and costs of US $\$ 14,600$ per QALY in 2001 dollars and US\$3,000 per QALY in 2005 dollars respectively. ${ }^{46,47}$

Few cost-benefit analyses on the HPV vaccine are available. One cost-benefit analysis performed on Medicaidenrolled females of the Appalachian region of Kentucky in the US found that utilizing the HPV vaccine as a primary route for prevention is indeed a cost-saving strategy when the rising cost of healthcare, aging population, and costs to treat cervical cancer are taken into account. The female population of the Appalachian region of Kentucky is underserved and particularly vulnerable to developing cervical cancer and dying from it due to a number of risk factors including poverty, lower education levels, barriers to health insurance and access to care, and higher smoking rates. ${ }^{48}$ The analysis found that though actual cost savings recognition would not occur for several decades as the vaccinated population ages, implementing a prevention plan for cervical cancer that includes annual HPV vaccination of Medicaid-enrolled adolescent females is not only beneficial to the public's health, but also a cost-efficient strategy to the Medicaid system. ${ }^{3}$

Mathematical models are a valuable asset because they can mimic the natural history of disease and provide information that integrates both clinical and economical data. This information is useful in policy decision-making as well as budget determinations in addition to estimating potential cost-effectiveness of multiple strategies. Since the HPV vaccine has been determined to be a cost-effective primary prevention strategy, care is necessary in formulating program budgets, especially in the initial years of vaccination, when the costs to vaccinate against HPV and treat cervical cancer are still high. Recognition of cost-savings will not occur immediately. Should implementation of immunization programs occur, it would not be until the population of vaccinated adolescent females aged 12 to 25 reach at least the median age for diagnosis of cervical cancer, which was 48 years during the 2000 to 2004 period.

The costs to treat cervical cancer cases in nonvaccinated females and the costs to maintain screening programs would still incur. However, consideration of the fact that the HPV vaccine will also decrease incidence of anogenital warts and other associated HPV diseases in addition to thwarting the need for diagnosis, treatment, and surgical intervention of these ailments is important to acknowledge and may offset additional costs. While only monetary costs are considered here, the HPV vaccine should decrease quality-of-life costs 
in all females who receive the vaccine. Though vaccinating against HPV may indeed be a cost-effective strategy, the realization of this effectiveness would not occur for another 25 to 30 years postimmunization program initiation, and consideration of these total direct and indirect costs attributable to vaccination and treatment of cervical cancer is necessary. ${ }^{49}$

\section{Implication for healthcare policy}

Healthcare costs are growing rapidly and the economic impact of HPV and cervical cancer is substantial. ${ }^{50}$ Expenditures of an estimated US\$4 billion occur in the US on direct costs associated with HPV, its sequela, and cervical cancer. Each year, almost US\$200 million is ascribable to managing anogenital warts, US\$300 to US\$400 million on invasive cervical cancer, and the residual funds on routine cervical cancer screening, follow-up of abnormal Pap tests, and cervical intraepithelial neoplasia. ${ }^{15,51}$ It is therefore imperative to streamline cervical cancer screening programs to make them more effective and efficient especially in the current ailing global economy.

Since the debut of the HPV vaccine, global acceptance has been slow for numerous reasons including the lack of awareness of HPV and its link to cervical cancer. ${ }^{52}$ Increasing public knowledge is critical as well as garnering support from physicians whose recommendations are substantial in influencing parents to get their children vaccinated if not mandated to do so. ${ }^{53}$ Despite the fact that the efficacy of both the quadrivalent and bivalent vaccines and demonstrated cost-effectiveness, other issues such as challenges in delivering the vaccine prevent worldwide recognition and acceptance of the HPV vaccine as well. One study found that reluctance to immunize depends on certain characteristics of the vaccine including cost and safety, insurance coverage, and the potential impact of the vaccine on adolescent female sexual activity. ${ }^{54}$ Dispelling misconceptions regarding the vaccine with education about the vaccine will support global attainment of maximal immunization coverage against HPV and increase invasive cervical cancer aversion.

Research indicates that the HPV vaccine will be beneficial in reducing the high-risk types of HPV infection, which account for $59 \%$ of total HPV infections and cause cervical cancer, as well as two of the low-risk types that are associated with genital warts in the US. ${ }^{42}$ Despite the fact that the vaccine is highly efficacious, most studies recommend retention of screening as a method for early detection. Instead, studies suggest delaying the age of onset of Pap screening until the mid-thirties for vaccinated individuals, since the vaccine prevents the primary types of HPV infections that are associated with cervical cancer and manifest in younger sexually active adolescents. This ensures that the practice is more efficient and less costly. ${ }^{55}$ In addition, recent research supports decreasing the frequency at which screening occurs in vaccinated females from annually to approximately every 3 years. This would further increase the cost-effectiveness of cervical cancer screening programs that require screening of large numbers of women in order to prevent one death when a majority of women $(80 \%)$ with high-grade cervical intraepithelial neoplasia do not progress to invasive cancer. ${ }^{55,56}$

A number of barriers exist for the women of low-income, resource poor communities. Barriers to optimal screening, diagnosis, and treatment of cervical cancer, including poverty, geographic isolation, isolation due to age, illiteracy, culture, fear, acceptability by healthcare providers, and lack of availability of community care. ${ }^{48}$ These barriers could similarly affect the number of women that receive the HPV vaccine. The vaccine, administered in a three-part process, requires at least three separate visits to a healthcare provider. These barriers, combined with the fact that generally fewer healthcare providers are available in impoverished areas that practice preventive medicine rather than treat acute conditions, can be a significant deterrent to women who may have to travel long distances to see a provider. Receiving education about HPV, cervical cancer, and the vaccine itself may depend on its availability, accessibility, and acceptability and could have a significant impact.

Public health efforts and policy should focus on maximizing HPV vaccine coverage by making it affordable and accessible to all women and educating the public on the benefits of the HPV vaccines. At present, legislature in the US and around the world has sought to install mandatory requirements for girls of middle school entrance age to receive the vaccine in order to minimize HPV rates and increase population immunity. Moreover, actualization of the largest scale of herd immunity against HPV would occur should the vaccine gain licensure and promotion for use in males.

\section{Conclusions}

The effects of the HPV vaccine, aside from prevention of persistent HPV infection, on cervical cancer are unascertainable at present. Administration of vaccination against HPV is recommended in young females age 12 to 25 , though the vaccine is safe for use among females aged 9 through $26 .{ }^{57}$ The cost of the three vaccine series, approximately US\$360, is the most expensive vaccine available to date. It poses a major barrier of access to many women including those most likely to benefit from the vaccine: 
females from low-income communities and communities of color. ${ }^{58}$ Despite the fact that public financing may be obtainable to those who qualify and the availability of the quadrivalent vaccine through a vaccine assistance program to some females that are uninsured, many do not meet these criteria or have insurance coverage by companies that do not pay for the immunization. These females may not have access to the vaccine because of its significant expense. ${ }^{59}$ Furthermore, neither is known of the longevity of immunity conferred by the vaccine nor the need for booster shots in the future to maintain immunity. If titers are necessary, the costs associated with vaccination against HPV will rise. ${ }^{42}$ Regardless, most studies maintain that administration of the vaccine should occur in females around the 12 th year of life. ${ }^{60}$

The predicted increase of quality-adjusted life expectancy for females of this age is 2.8 days or 4.0 quality-adjusted life-days. The vaccine, it is further hypothesized, will avert more than 224,255 cases of HPV infection, 112,710 cases of squamous intraepithelial lesions, 3317 cases of cervical cancer, and 1340 deaths caused by cervical cancer over the life span of the present US cohort of 12-year-old females. Moreover, in order to avert or prevent one case of cervical cancer, 600 girls would require vaccination. ${ }^{42}$

Cost-related studies reinforce the importance of such economic analyses in determining resource allocation, especially in public health, and further support evidence-based decision-making when considering public health interventions and other prevention programs. Further research should reevaluate cervical cancer screening recommendations, vaccine administration, and program implementation. Additional investigation of the duration of protection from both the bivalent and quadrivalent vaccines, virologic surveillance, and the safety of the HPV vaccines should continue in the future. Monitoring HPV-related outcomes and exploring the possibility of vaccinating against HPV at the same time as other adolescent vaccines is of significant value. Consideration of male immunization and herd immunity will also affect future public health policy.

\section{Disclosures}

The author has no conflicts of interest to declare.

\section{References}

1. Foerster V, Murtagh J. Vaccines for prevention of human papillomavirus infection. Issues in emerging health technologies, Issue 75. Ottawa: Canadian Coordinating Office for Health Technology Assessment; 2005.

2. Saslow D, Castle P, Cox JT, et al. American Cancer Society Guideline for Human Papillomavirus (HPV) Vaccine Use to Prevent Cervical Cancer and Its Precursors. CA Cancer J Clin. 2007;57(1):7-28.
3. Prasad S, Hill R. A Cost-Benefit Analysis on the HPV Vaccine in Medicaid-Enrolled Females of the Applachian Region of Kentucky. KMA. 2008;106:271-276.

4. Torné A, Alonso I, Puig-Tintoré, Pahisa J. Clinical role of cervical cancer vaccination: When and whom to vaccinate? Gynecol Oncol. 2008;110:S15-S16.

5. Bosch FX, Lorincz A, Muñoz N, Meijer CJ, Shah KV. The causal relation between human Papillomavirus and cervical cancer. $J$ Clin Pathol. 2002;55:244-265.

6. Dasbach EJ, Elbasha EH, Insinga RP. Mathematical Models for Predicting the Epidemiologic and Economic Impact of Vaccination against Human Papillomavirus Infection and Disease. Epidemiol Rev. 2006;28:88-100.

7. Moscicki A. Epidemiology and oncogenesis of human papillomavirus (HPV) infection. In Human Papillomavirus Vaccines: Future Role of the Pediatrician in Preventing Cervical Cancer. New York, NY: The Academy for Healthcare Education; 2005. p. 1-3.

8. Chesson HW, Ekwueme DU, Saraiya M, Markowitz L. Cost-effectiveness of human papillomavirus vaccination in the United States. Emerg Infect Dis. 2008;14:244-251.

9. Parkin DM, Bray F. Chapter 2: the burden of HPV-related cancers. Vaccine. 2006;24(Suppl 3):S11-S25.

10. Centers for Disease Control and Prevention. Quadrivalent human Papillomavirus vaccine: recommendation of the Advisory Committee on Immunization Practices (ACIP). MMWR Recomm Rep. 2007;56 (RR-2):1-24.

11. Clifford GM, Smith JS, Aguado T, Franceschi S. Comparison of HPV type distribution in high-grade cervical lesions and cervical cancer: a meta-analysis. Br J Cancer. 2003;89:101-105.

12. Dunne EF, Markowitz LE. Genital human papillomavirus infection. Clin Infect Dis. 2006;43:624-629.

13. Greer CE, Wheeler CM, Ladner MB, et al. Human papillomavirus (HPV) type distribution and serological response to HPV type 6 virus-like particles in patients with genital wars. J Clin Microbiol. 1995;33:2058-2063.

14. Chesson HW, Blandford JM, Gift TL, Tao G, Irwin KL. The estimated direct medical cost of sexually transmitted diseases among American youth, 2000. Perspect Sex Reprod Health. 2004;36: $11-19$.

15. Insinga RP, Dasbach EJ, Elbasha EH. Assessing the annual economic burden of preventing and treating anogenital human papillomavirusrelated disease in the US: analytic framework and review of the literature. PharmacoEconomics. 2005;23:1107-1122.

16. Franco E, Harper D. Vaccination against human Papillomavirus infection: a new paradigm in cervical cancer control. Vaccine. 2005;23:2388-2394.

17. Blatter MM. HPV Vaccine Efficacy and Tolerability. In Human Papillomavirus Vaccines: Future Role of the Pediatrician in Preventing Cervical Cancer. New York, NY: The Academy for Healthcare Education; 2005. p. 6-7.

18. Saslow D, Runowicz CD, Solomon D, et al. American Cancer Society guideline for the early detection of cervical neoplasia and cancer. $C A$ Cancer J Clin. 2002;52:342-362.

19. International Agency for Research on Cancer (IARC). Handbooks of Cancer Prevention: Cervix Cancer Screening. Lyon, France: IARC; 2005.

20. Devesa SS. Descriptive epidemiology of cancer of the uterine cervix. Obstet Gyencol. 1984;63:605-612.

21. Ries L, Harkins D, Krapcho M, et al. SEER Cancer Statistics Review, 1975-2003: National Cancer Institute. Bethesda, MD: National Cancer Institute; 2006.

22. Rogoza RM, Ferko N, Bentley J, et al. Optimization of primary and secondary cervical cancer prevention strategies in an era of cervical cancer vaccination: A multi-regional health economic analysis. Vaccine. 2008;26S:F46-F58.

23. Goldie SJ, Kim JJ, Myers E. Chapter 19: cost-effectiveness of cervical cancer screening. Vaccine. 2006;24(S3):164-170. 
24. Sawaya GF, Washing AE. Cervical cancer screening: which techniques should be used and why? Clin Obstet Gynecol. 1999;42:922-938.

25. Nanda K, McCrory DC, Myers ER, et al. Accuracy of the Papanicolaou test in screening for and follow-up of cervical cytologic abnormalities: a systemic review. Ann Intern Med. 2000;132:810-819.

26. Stoler MH, Schiffman M. Atypical Squamous Cells of Undetermined Significance-Low grade Squamous Intraepithelial Lesion Triage Study (ALTS) Group. Interobserver reproducibility of cervical cytologic and histologic interpretations: realistic estimates from the ASCUS-LSIL Triage Study. JAMA. 2001;285:1500-1505.

27. Denny L, Kuhn L, De Souza M, Pollack A, Dupree W, Wright T. Screenand-Treat Approaches for Cervical Cancer Prevention in Low-Resource Settings: A Randomized Controlled Trial. JAMA. 2005;294:2173-2181.

28. Wright TC Jr, Cox JT, Massad LS, Twiggs LB, Wilkinson EJ. 2001 consensus guidelines for the management of women with cervical cytological abnormalities. JAMA. 2002;287:2120-2129.

29. Bayas J, Costas L, Muñoz A. Cervical cancer vaccination indications, efficacy, and side effects. Gynecol Oncol. 2008;110:S11-S14

30. Pinto LA, Edwards J, Castle PE, et al. Cellular immune responses to human papillomavirus (HPV)-16 L1 in healthy volunteers immunized with recombinant HPV-16 L1virus-like particles. J Infect Dis. 2003;188:327-338.

31. Emeny RT, Wheeler CM, Jansen KU, et al. Priming of human papillomavirus type 11-specific humoral and cellular immune responses in college-aged women with a virus-like particle vaccine. $J$ Virol. 2002;76:7832-7842.

32. Villa LL, Costa RL, Petta CA, et al. Prophylactic quadrivalent human papillomavirus (types 6, 11, 16, and 18) L1 virus-like particle vaccine in young women: a randomized double-blind placebo-controlled multicentre phase II efficacy trial. Lancet Oncol. 2005;6:271-278.

33. Villa LL, Ault KA, Giuliano AR, et al. Immunologic responses following administration of a vaccine targeting human papillomavirus Types 6, 11, 16, 18. Vaccine. 2006;24:5571-5583.

34. Villa LL, Costa RL, Petta CA, et al. High sustained efficacy of a prophylactic quadrivalent human papillomavirus types 6/11/16/18 L1virus like particle vaccine through 5 years of follow-up. Br J Cancer. 2006;95:1459-1466.

35. Blatter MM. HPV Vaccine Efficacy and Tolerability. In Human Papillomavirus Vaccines: Future Role of the Pediatrician in Preventing Cervical Cancer. New York, NY: The Academy for Healthcare Education; 2005. p. 6-7.

36. Keam S, Harper D. Human Papillomavirus Types 16 and 18 Vaccine (Recombinant, AS04 Adjuvanted, Adsorbed) [Cervarix ${ }^{\mathrm{TM}}$ ]. Drugs. 2008;68:359-372.

37. GlaxoSmithKline. GSK submits Cervarix ${ }^{\mathrm{TM}}$ license application to Japan's health authorities [media release]. 2007 Sept 28.

38. Harper DM, Franco EL, Wheeler CM, et al. Sustained efficacy up to 4.5 years of a bivalent L1 virus-like particle vaccine against human papillomavirus types 16 and 18: follow-up from a randomized control trial. Lancet. 2006;367:1247-1255.

39. Bouchardy C, Rapiti E, Blagojevic S, Vlastos A, Vlastos G. Older Female Cancer Patients: Importance, Causes, and Consequences of Undertreatment. J Clin Oncol. 2005;25:1858-1869.

40. Brownson R, Baker E, Leet T, Gillespie K. Evidence-Based Public Health. Oxford University Press, Inc: New York, New York; 2005.
41. Insigna R, Glass A, Rush B. The health care costs of cervical human papillomavirus-related disease. Am J Obste Gynecol. 2004;191:114-120.

42. Sanders GD, Taira AV. Cost Effectiveness of a Potential Vaccine for Human Papillomavirus. Emerg Infect Dis. 2003;9:37-48.

43. Myers ER, McCrory DC, Nanda K, Bastian L, Matchar DB. Mathematical model for the natural history of human papillomavirus infection and cervical carcinogenesis. Am J Epidemiol. 2000;151:1158-1171.

44. Dasbach EJ, Elbasha EH, Insinga RP. Mathematical models for predicting the epidemiologic and economic impact of vaccination against human papillomavirus infection and disease. Epidemiol Rev. 2006;28:88-100.

45. Shi L. Health Services Research Methods. Albany, NY: Delmar Thompson Learning; 1997.

46. Taira AV, Neukermans CP, Sanders GD. Evaluating human papillomavirus vaccination programs. Emerg Infect Dis. 2004;10:1915-1923.

47. Elbasha E, Dasbach EJ, Insinga RP. Model for assessing human papillomavirus vaccination. Emerg Infect Dis. 2007;13:29-41.

48. Friedell G, Linville L, Hullet S. Cancer control in rural Appalachia. In Cancer Supplement, 6th Biennial Symposium on Minorities, the Medically Underserved and Cancer. 1998;83:1868-1871.

49. Surveillance Epidemiology and End Results (SEER) [homepage on the Internet]. Cancer Stat Fact Sheets: Cancer of the Cervix Uteri. [cited 2003 Feb 13]. Available from: http://seer.cancer.gov/statfacts/html/ cervix_print.html

50. Bodenheimer T. High and Rising Health Care Costs. Part 1: Seeking an Explanation. Ann Internal Med. 2005;42:847-854.

51. Chesson JM, Blandford TL, Gift GT, Irwin KL. The Estimated Direct Medical Cost of Sexually Transmitted Diseases Among American Youth, 2000. Perspect Sex Reprod Health. 2004;36:11-19.

52. Tiro J, Meissner H, Kobrin S, et al. What Do US Women Know About HPV and Cervical Cancer? Cancer Epidemiol Biomarkers Prev. 2007;16:288-294.

53. Zimet G. Improving Adolescent Health: Focus on HPV Vaccine Acceptance. J Adolesc Health. 2005;37:S17-S23.

54. Ishibashi K, Koopmans J, Curlin F, Alexander K, Ross L. Paediatricians' attitudes and practices towards HPV vaccination. Acta Paediatric. 2008;97:1550-1556.

55. Kulasingam SL, Myers ER. Potential Health and Economic Impact of Adding a Human Papillomavirus Vaccine to Screening Programs. JAMA. 2003;290:781-789.

56. Raffle AE, Alden BA, Quinn M, Babb PJ, Brett MT. Outcomes of screening to prevent cancer: analysis of cumulative incidence of cervical abnormality and modeling of cases and deaths prevented. $\mathrm{Br} \mathrm{Med} J$. 2003;326:901-905.

57. Centers for Disease Control and Prevention (CDC). Recommended Immunization Schedules for Persons Aged 0-18 Years - United States, 2007. MMWR. 2007;55:Q1-Q4

58. Dailard C. Achieving Universal Vaccination Against Cervical Cancer In the United States: The Need and the Means. Guttmacher Policy Review. 2006;9:12-6

59. Merck Vaccine Patient Assistance Program. Merck and Co., Inc. 2008. Available at: http://www.merck.com/merckhelps/vaccines/home.html. Accessed April 30, 2009.

60. Dunne EF, Unger ER, Sternberg M, McQuillan et al. Prevalence of HPV Infection Among Females in the United States. JAMA. 2007;297:813-819.
ClinicoEconomics and Outcomes Research

\section{Publish your work in this journal}

ClinicoEconomics \& Outcomes Research is an international, peerreviewed open-access journal focusing on Health Technology Assessment, Pharmacoeconomics and Outcomes Research in the areas of diagnosis, medical devices, and clinical, surgical and pharmacological intervention. The economic impact of health policy and health systems

\section{Dovepress}

organization also constitute important areas of coverage. The manuscript management system is completely online and includes a very quick and fair peer-review system, which is all easy to use. Visit http://www.dovepress.com/testimonials.php to read real quotes from published authors. 